\title{
An accurate empirical formula for determining the density of heat-resistant nickel alloys
}

\author{
D. A. Tarasov ${ }^{\dagger}$, O. B. Milder, A. G. Tyagunov \\ †datarasov@yandex.ru
}

Ural Federal University n. a. the first President of Russia B. N. Yeltsyn, Yekaterinburg, 620002, Russia

\begin{abstract}
The density of a substance is one of its main physical characteristics. This is especially true for materials used in aviation, where the mass of each structural element should be minimized as much as possible. When developing new structural materials, for example, heat-resistant nickel alloys, which are widely used in the manufacture of gas turbine engine parts, it is extremely important to have a reliable and accurate method for assessing the density of the material being developed. Until now, no unified method has been proposed for calculating the density of heat-resistant nickel alloys. The paper reviews the available approaches to assessing the density of alloys and proposes a new formula that allows one to calculate the density of an alloy with a high accuracy based on the information on its composition. The proposed approach takes into account the spatial fcc structure of heat-resistant nickel alloys as well as the molar mass and molar volume of the elements that form the alloy. To check the accuracy of the calculations, a database of 69 heat-resistant nickel alloys was collected, containing information on the composition of the alloys and their known density. According to the proposed formula, as well as using some other known approaches, the density for the alloys from the database was calculated. The calculation results showed that the proposed method provided the best accuracy among all considered ones: the standard deviation of the calculated values from the real ones for the entire sample was $0.1 \%$, the mean values and medians practically coincide. In addition, the calculation errors are normally distributed and have an average value of -0.0001 . The existing methods give a minimum error of $1.2 \%$, thus, the proposed approach improved the accuracy of calculating the density of heat-resistant nickel alloys by about an order of magnitude, which is a significant result both from the point of view of the general scientific approach and from the point of view of engineering practice. Taking into account the results obtained, the proposed formula can be widely used in the development of new and modification of existing heat-resistant nickel alloys.
\end{abstract}

Keywords: heat-resistant nickel alloys, mechanical properties, composition, density.

УДК: 669.245

\section{Точная эмпирическая формула определения плотности жаропрочных никелевых сплавов}

\footnotetext{
Тарасов Д. А. ${ }^{\dagger}$, Мильдер О. Б., Тягунов А.Г.

Уральский федеральный университет имени первого Президента России Б.Н. Ельцина, Екатеринбург, 620002, Россия

Плотность вещества является одной из его основных физических характеристик. Особенно это касается материалов, использующихся в авиации, где масса каждого элемента конструкции должна по возможности минимизироваться. При разработке новых конструкционных материалов, например, жаропрочных никелевых сплавов, широко применяющихся при изготовлении деталей газотурбинных двигателей, чрезвычайно важно иметь надежный и точный метод оценки плотности разрабатываемого материала. До настоящего времени не предложено единого метода расчета плотности жаропрочных никелевых сплавов. В работе проводится обзор имеющихся подходов к оценке плотности сплавов и предлагается новая формула, позволяющая с высокой точностью рассчитать плотность сплава на основании информации о его составе. Предложенный подход учитывает пространственную ГЦК-структуру жаропрочных никелевых сплавов, а также молярную массу и молярный объем элементов, составляющих сплав. Для проверки точности расчетов была собрана база данных из 69 жаропрочных никелевых
} 
сплавов, содержащая информацию о составе сплавов и их известной плотности. По предложенной формуле, а также при помощи некоторых других известных подходов были рассчитаны плотности сплавов из базы данных. Результаты расчетов показали, что предложенная методика обеспечивает наилучшую точность из всех рассмотренных: среднеквадратичное отклонение расчетных значений от реальных по всей выборке составило $0.1 \%$, средние значения и медианы практически совпадали. Кроме того, ошибки расчета распределены нормально и имеют среднее значение -0.0001. Существующие методы дают минимальную ошибку 1.2\%, таким образом, предложенный подход приблизительно на порядок улучшил точность расчета плотности жаропрочных никелевых сплавов, что является существенным результатом как с точки зрения общенаучного подхода, так и с точки зрения инженерной практики. С учетом полученных результатов, предложенная формула может быть широко использована при разработке новых и модификации существующих жаропрочных никелевых сплавов.

Ключевые слова: жаропрочные никелевые сплавы, механические свойства, состав, плотность.

\section{1. Введение}

Разработка жаропрочных никелевых сплавов (ЖНС) со специальными свойствами для применения в газотурбинных двигателях является сложной научной и инженерной задачей, требующей высокой концентрации усилий специалистов, особенно в свете тенденций на снижение плотности сплавов с сохранением и улучшением их характеристик и применение высокототехнологичного монокристаллического литья [1-4].

При выплавке ЖНС широко используются лиганды с большим атомным весом, такие как вольфрам, молибден, тантал, рений, что приводит к повышению плотности сплавов и, как следствие, увеличению массы изделий из них. Поскольку одной из основных областей применения ЖНС является авиация, вопрос веса той или иной детали является чрезвычайно важным, а иногда - критическим. Современные принципы экономного легирования в первую очередь ставят своей целью снижение стоимости сплава, но параллельно с этим рассматриваются и вопросы снижения плотности, в частности за счет снижения содержания дорогостоящего и чрезвычайно плотного рения [5-7].

Влияние состава сплавов на их механические свойства является одним из основных предметов исследований. Плотность сплава, очевидно, напрямую связана с его составом и микроструктурой $[8,9]$. В свете вышесказанного было бы полезно при разработке ЖНС количественно учитывать фактор плотности, а для этого необходимо иметь метод точной ее оценки, а именно аналитическую зависимость плотности сплава от содержания в нем легирующих элементов.

Необходимо также разделять вопросы оценки плотности сплавов в твердом и жидком состоянии. Поскольку гетерофазные расплавы из-за фазовых переходов представляют собой структуры, демонстрирующие существенную нелинейность параметров [10-12], значения плотности расплавов также немонотонно изменяются с температурой, проявляя экстремумы при фазовых превращениях металлических жидкостей. Эти вопросы находятся за рамками этой работы. Предметом нашего исследования является плотность ЖНС при нормальных условиях, для определения которой мы связали физические принципы вычисления плотности твердых веществ и эмпирический подход, основанный на учете особенностей структуры ЖНС.

\section{2. Структура и подходы к оценке плотности ЖНС}

Принято считать, что структура ЖНС представляет собой $\gamma$-твердый раствор легирующих элементов в никелевой матрице (геометрическая модель - ГЦК-структура [13]) и дисперсных выделений $\gamma^{\prime}$-фазы на основе $\mathrm{Ni}_{3} \mathrm{Al}$ при очень близких периодах их кристаллических решеток, дающих взаимное несоответствие («мисфит») не выше $0.5 \%$ и результирующим параметром ячейки решетки, рассчитывающимся как арифметическое среднее параметров решеток $\gamma$ - и $\gamma^{\prime}$-фаз. Этот фактор необходимо в той или иной степени учесть при расчетах плотности ЖНС. Стоит отметить, что при экстремальных давлениях (десятки ГПа) ГЦК-решетки могут трансформироваться в ОЦК и ГПУ [14], однако при работе ЖНС такие режимы не достигаются, поэтому все сплавы считаются основанными на ГЦК-геометрии.

Плотность простого вещества определяется как отношение массы $d m$ элемента тела к объему $d V$ этого элемента (1). Модификацией формулы (1) является предложенное в [15, с. 246] выражение (2), где $n$ - число атомов в элементарной ячейке, $V$ - объем элементарной ячейки $\left(\mathrm{cm}^{3}\right), A-$ атомная масса вещества, а численный множитель есть коэффициент пересчета из атомных единиц массы (а.е.м.) в граммы.

$$
\begin{gathered}
\rho=\frac{d m}{d V}, \\
\rho=\frac{n A \cdot 1.66 \cdot 10^{-24}}{V} .
\end{gathered}
$$

Нам представляется более логичным использовать промежуточный вариант между (1) и (2), а именно подход на основе молярных соотношений (3), где $\mu-$ это молярная масса вещества (г/моль), а $v-$ молярный объем ( $\mathrm{cm}^{3} /$ моль).

$$
\rho=\frac{\mu}{v} .
$$

Использование формулы (3) представляется оптимальным по следующим соображениям. Молярная масса учитывает усредненный изотопный состав, ее значение выражается в г/моль, что соответствует массовому числу элемента в таблице Менделеева. К настоящему моменту значения молярных масс $d$-переходных металлов, состав- 
ляющих основу ЖНС, известны с высокой степенью точности. Значения молярных объемов имеют по крайней мере три значащие цифры и могут быть взяты, например, в [15, с. 247,16].

При оценке плотности ЖНС традиционно используется несколько базовых подходов. Назовем их условно «аналитический», «регрессионный» и «метод средней плотности». Аналитический подход учитывает тот факт, что ЖНС являются твердыми растворами замещения и упаковываются в ГЦК-решетку никелевой матрицы. С использованием (3) плотность сплава может быть оценена при помощи выражения (4), где $\bar{\mu}=\sum_{i} \mu_{i} n_{i}$, $\bar{\mu}$ - это молярная масса $i$-го компонента сплава, $v_{(\mathrm{Ni})}=6.59 \mathrm{~cm}^{3} /$ моль - молярный объем никеля, $n_{i}-$ атомная доля $i$-го компонента, при этом имеет место очевидная нормировка $\sum_{i} n_{i}=1$.

$$
\rho=\frac{\bar{\mu}}{v_{(\mathrm{Ni})}}=\frac{\sum_{i} \mu_{i} n_{i}}{v_{(\mathrm{Ni})}} .
$$

Необходимо обратить отдельное внимание на переход от традиционно применяемых в описании ЖНС весовых долей компонентов сплава к атомным, которая осуществляется по формуле (5), где $n_{\text {wti }}-$ весовая доля $i$-го компонента сплава. По нашему мнению, использование атомных долей содержания легирующих элементов вместо весовых при описании содержания ЖНС более точно характеризует картину распределения элементов с учетом их атомных характеристик. В частности, при описании соединений стехиометрического состава, например, $\gamma^{\prime}-\mathrm{Ni}_{3} \mathrm{Al}$, соотношение $n_{i}(\mathrm{Ni}) / n_{i}(\mathrm{Al})$ в точности составляет $3: 1$, тогда как соотношение весовых концентраций будет сдвинуто в сторону никеля, как более плотного компонента.

$$
n_{i}=\frac{n_{\mathrm{wt} i} / \mu_{i}}{\sum_{i} \frac{n_{\mathrm{wt} i}}{\mu_{i}}}
$$

Были предприняты попытки модифицировать формулу (4) таким образом, чтобы учесть мисфит матрицы $\gamma$-твердого раствора и дисперсных выделений $\gamma^{\prime}$-фазы [17]. Авторы использовали простое арифметическое среднее параметров решетки, хотя с нашей точки зрения логичней было бы включить в расчет информацию об объемной доле $\gamma^{\prime}$-фазы в конкретном сплаве. Кроме того, авторы указывают, что мисфит редко превышает $0.5 \%$, что в пересчете на объем элементарной ячейки дает поправку к расчетному значению плотности в $1.5 \%$.

Другим подходом к оценке плотности ЖНС является построение многомерной регрессии [18-20], которая строится по отношению к весовым долям компонентов сплава. Это демонстрирует тот же традиционный, но ошибочный подход описания микро-свойств (атомный уровень) при помощи макро-характеристик (массы компонентов). Более того, как во всякой подобной модели, значение коэффициентов регрессии сильно зависит от состава и объема выборки, на основании данных которой регрессия строилась. Заявленная погрешность модели [18] составляет $1.2 \%$.
Третий подход к оценке плотности сплавов основан на расчете средней плотности простых металлов - компонентов сплава (6), где $\rho_{i}-$ плотность $i$-го «чистого» компонента сплава, $n_{i}$ - атомная доля.

$$
\rho=\sum_{i} \rho_{i} n_{i}, \quad \sum_{i} n_{i}=1,
$$

Методы оценки плотности по формуле (6), часто реализованы в виде онлайн калькуляторов [21]. Поскольку данный подход является менее физически обоснованным по сравнению с аналитическим, он дает, как правило, большую погрешность «с недостатком», так как не учитывает способ упаковки результирующего сплава.

Существуют и комбинированные подходы. Так в [22] автор предложил формулу расчета плотности ЖНС (7), учитывающую одновременно среднюю плотность компонент сплава и эмпирическую регрессионную добавку, где $n_{\mathrm{wt} i}-$ весовая доля $i$-го компонента сплава, $\rho_{i}-$ плотность $i$-го компонента сплава, $k_{i}, l_{1}$ и $l_{2}-$ коэффициенты регрессии при квадратичных членах, соответствующим концентрации молибдена и кобальта (см. Табл. 1). Мы пересчитали для удобства читателя размерность фунт/дюйм ${ }^{3}$ в более привычные г/ $\mathrm{cm}^{3}$ и привели все коэффициенты с учетом пересчета в Табл. 1.

$$
\begin{aligned}
\rho= & \frac{100}{\sum_{i} \frac{n_{\mathrm{wt} i}}{\rho_{i}}}+3.88543+ \\
+ & \sum_{i} n_{\mathrm{wt} i} k_{i}+l_{1} n_{\mathrm{wt}(\mathrm{Mo})}{ }^{2}+l_{2} n_{\mathrm{wt}(\mathrm{Co})} \cdot n_{\mathrm{wt}(\mathrm{Ti})} .
\end{aligned}
$$

Автор традиционно оценивал количественные соотношения элементов в сплаве на основе данных об их весовых долях (точнее, весовых процентах, wt.\%), а не атомных, как того требует подход. Кроме того, в регрессионной части не учтено влияние таких элементов, как алюминий, гафний, цирконий, рений, рутений.

Можно сравнить эти результаты с параметрами модели, описанной в [18], где, в частности, используется только регрессия (8) с соответствующими коэффициентами, приведенными в Табл. 2.

$$
\rho=8.531+\sum_{i} n_{\mathrm{wt} i} k_{i} .
$$

Можно отметить отсутствие согласования между этими моделями, особенно в части оценки вклада

Табл. 1. Параметры модели Халла [22].

Table 1. The Hull [22] model parameters.

\begin{tabular}{|c|c|c|c|c|}
\hline$i$ & $\begin{array}{c}\text { Элемент } \\
\text { Element }\end{array}$ & $\begin{array}{c}\text { Плотность } \rho_{i}, \mathrm{r} / \mathrm{cm}^{3} \\
\text { Density, } \rho_{i}, \mathrm{~g} / \mathrm{cm}^{3}\end{array}$ & $k_{i}$ & $l_{i}$ \\
\hline 1 & $\mathrm{Cr}$ & 7.1900 & -0.03792 & $5.17614 \times 10^{-4}$ \\
\hline 2 & $\mathrm{Ni}$ & 8.9081 & -0.03848 & $-0.00140 \times 10^{-4}$ \\
\hline 3 & $\mathrm{Co}$ & 8.9023 & -0.03931 & \\
\hline 4 & $\mathrm{Fe}$ & 7.8740 & -0.03875 & \\
\hline 5 & $\mathrm{Mo}$ & 10.2796 & -0.05148 & \\
\hline 6 & $\mathrm{~W}$ & 19.2501 & -0.03460 & \\
\hline 7 & $\mathrm{~V}$ & 6.1100 & -0.03709 & \\
\hline 8 & $\mathrm{Nb}$ & 8.5699 & -0.03294 & \\
\hline 9 & $\mathrm{Ta}$ & 16.6501 & -0.03128 & \\
\hline 10 & $\mathrm{Ti}$ & 4.5068 & 0.01107 & \\
\hline 11 & $\mathrm{C}$ & 2.2600 & 0.10740 & \\
\hline
\end{tabular}


Табл. 2. Параметры модели [18].

Table 2. The model parameters from [18].

\begin{tabular}{|c|c|c|}
\hline$i$ & Элемент / Element & $k_{i}$ \\
\hline 1 & $\mathrm{C}$ & -0.419 \\
\hline 2 & $\mathrm{Cr}$ & -0.009 \\
\hline 3 & $\mathrm{Co}$ & -0.002 \\
\hline 4 & $\mathrm{~W}$ & 0.055 \\
\hline 5 & $\mathrm{Mo}$ & 0.026 \\
\hline 6 & $\mathrm{Ti}$ & -0.045 \\
\hline 7 & $\mathrm{Al}$ & -0.093 \\
\hline 8 & $\mathrm{Nb}$ & 0.023 \\
\hline 9 & $\mathrm{Ta}$ & 0.049 \\
\hline 10 & $\mathrm{Hf}$ & 0.022 \\
\hline 11 & $\mathrm{Re}$ & 0.059 \\
\hline 12 & $\mathrm{Ru}$ & 0.013 \\
\hline & &
\end{tabular}

отдельных элементов в плотность сплава. Таким образом, можно констатировать, что какой-либо единой регрессионной модели до сих пор не создано. Сложности добавляет тот факт, что различные авторы предлагают учитывать различные наборы элементов, формирующих сплавы. Таким образом, мы считаем регрессионные подходы слишком специализированными, тогда как инженерная практика требует более универсальных методов.

\section{3. Описание подхода и экспериментальная проверка}

Мы оттолкнулись от аналитического подхода (4), объединяющего молярные массы и молярные объемы компонентов сплава. Предварительный расчет показал, что формула (4) дает оценку плотности «с избытком». Очевидно, что молярный объем реальных сплавов претерпевает определенные изменения при взаимодействии никеля и легирующих элементов, поэтому использование молярного объема чистого никеля в знаменателе формулы (4) требует введения поправочного коэффициента $k$, связанного с набором и относительной атомной долей легирующих элементов.

Таким образом, запишем выражение для плотности в виде (9), где коэффициент $k$ определяется по формуле (10):

$$
\begin{gathered}
\rho=\frac{\bar{\mu}}{k v_{(\mathrm{Ni})}}=\frac{\sum_{i} \mu_{i} n_{i}}{k v_{(\mathrm{Ni})}}, \\
k=\prod_{i}\left(\frac{v_{i}}{v_{(\mathrm{Ni})}}\right)^{\frac{n_{i}}{2}} .
\end{gathered}
$$

В формуле (9) не учитываются такие легкие элементы, как бор, углерод, фосфор, сера и прочие, в силу того, что в $\gamma$-твердом растворе они являются элементами внедрения, а не замещения. Справочные физические характеристики никеля и легирующих элементов приведены по данным [16] в Табл. S1 (дополнительный материал).

Поправочный коэффициент $k$ имеет простой физический смысл: каждый легирующий элемент изменяет молярный объем сплава в той мере, в какой молярный объем чистого легирующего элемента относится к молярному объему чистого никеля, а степень этого влияния пропорциональна атомной доле этого элемента в составе сплава. Делитель 2 в показателе степени формулы (10) вводится вследствие того, что элементарная ГЦК-ячейка содержит 2 атома, то есть каждый замещающий атом легирующего элемента формально оказывает влияние в среднем на половину объема элементарной ГЦК-ячейки.

Дальнейшее уточнение формулы показало, что делитель в показателе степени является варьируемым параметром модели и требует подбора для получения оптимального результата. Наилучшая точность достигается при его значении 1.55. Таким образом, итоговую формулу расчета плотности ЖНС можно записать в виде (11):

$$
\rho=\frac{\sum_{i} \mu_{i} n_{i}}{v_{(\mathrm{Ni})} \prod_{i}\left(\frac{v_{i}}{v_{(\mathrm{Ni})}}\right)^{\frac{n_{i}}{1.55}}} .
$$

Для экспериментальной проверки точности расчета мы собрали базу данных, содержащую информацию о 69 ЖНС с точно определенным составом и измеренной плотностью (см. Табл. S2 (дополнительный материал)). Для сплавов из этой базы мы последовательно применили расчет плотности по формуле (11) и формулам (7) и (8). Результаты расчетов сравнили между собой и с данными объективно измеренных физическими методами плотностей. Ошибки при расчетах по предложенной нами формуле дополнительно оценили статистически.

\section{4. Результаты и обсуждение}

Сводная таблица результатов расчетов плотности сплавов по различным методикам приведена в дополнительном материале (см. Табл. S2). Диаграмма рассеяния расчетных и реальных значений плотности и диаграмма размаха приведены на Рис. 1а. Обе диаграммы указывают на хорошее согласование расчетных и экспериментальных значений плотности. Статистические параметры ошибки расчетов по формуле (11): медиана -0.0011 , среднее -0.0010 , макс. 0.2135, мин. -0.1775 , среднеквадратичное отклонение (СКО) 0.0705, средняя относительная ошибка 0.0061, относительная среднеквадратическая ошибка 0.0057. Сводные данные по результатам расчетов по формулам (11), (8) и (7) приведены в Табл. 3.

Дальнейший статистический анализ показал, что распределение ошибок близко к нормальному и имеет практически нулевое $(-0.0001)$ среднее, а 95\% ошибок расчета (по модулю) не превышают 0.16 г/ $\mathrm{cm}^{3}$ (см. Рис. 1b), что является чрезвычайно низким значением, особенно для практических инженерных задач.

Таким образом, предложенная формула (11), наряду с результатами регрессионной модели [5], обеспечила наилучшую расчетную точность - средняя ошибка $0.1 \%$. Однако в отличие от регрессионных методов физически обоснованная аналитическая модель (11) имеет всего один подбираемый параметр - делитель в показателе степени, что делает ее удобной для формализации, например, в рамках пакетов автоматизированных расчетов. 


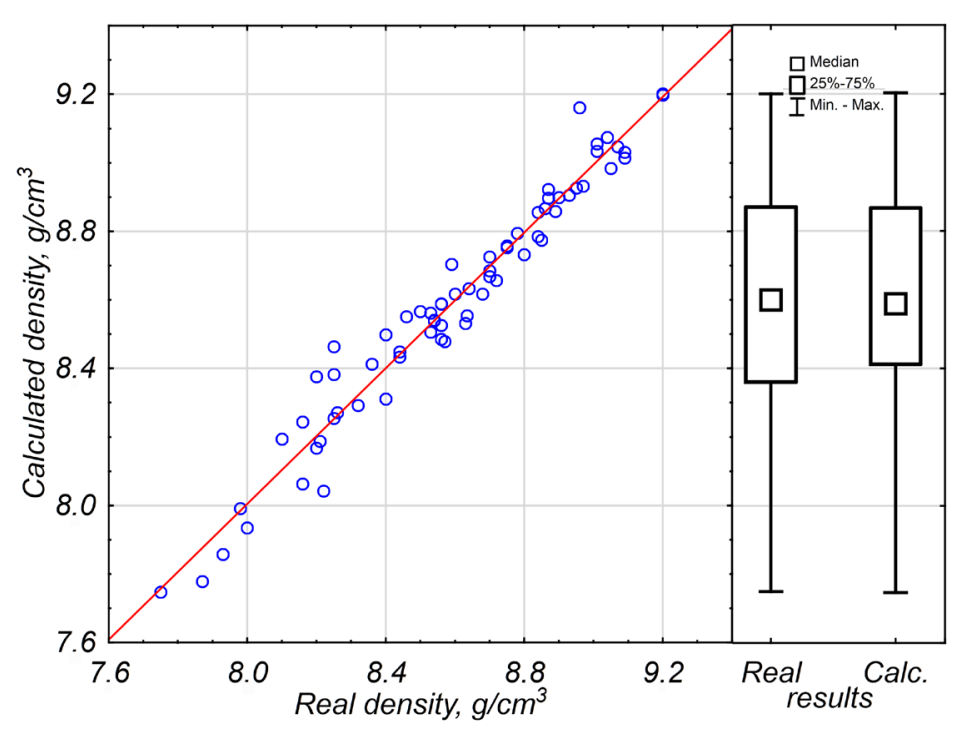

a

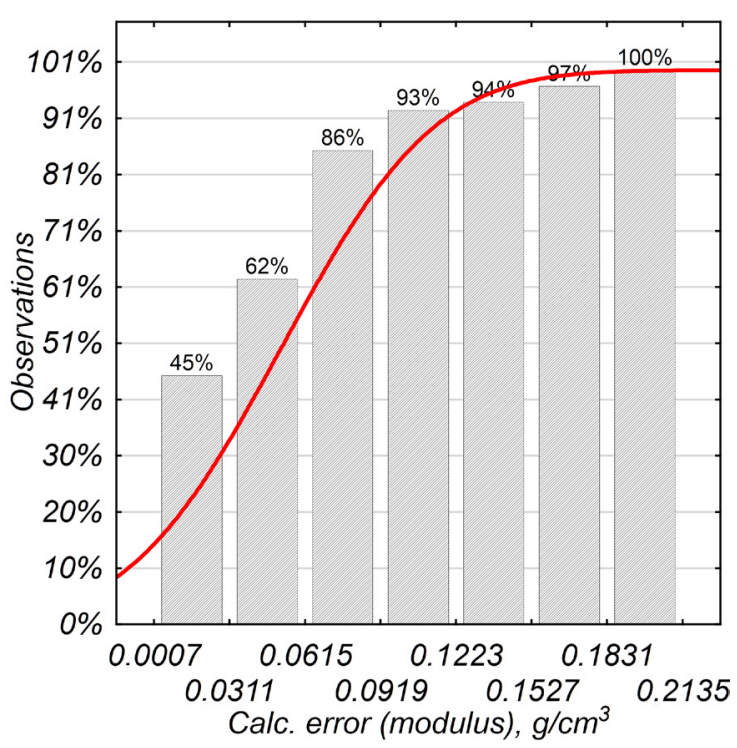

$\mathrm{b}$

Рис. 1. Результаты расчетов по предложенной формуле в сравнении с реальными плотностями сплавов: диаграмма рассеяния / диаграмма размаха (a); кумулятивное распределение модуля расчетных ошибок (b).

Fig. 1. The results of calculations using the proposed formula in comparison with the real densities of the alloys: scatter diagram / swing diagram (a); cumulative distribution of the calculation error modulus (b).

Табл. 3. Сравнение точности расчета плотности по различным формулам.

Table 3. Comparison of density calculation accuracy of different equations.

\begin{tabular}{|c|c|c|c|c|c|c|}
\hline $\begin{array}{c}\text { Расчетная формула } \\
\text { Calculation equation }\end{array}$ & $\begin{array}{c}\text { Медиана } \\
\text { Median }\end{array}$ & $\begin{array}{c}\text { Среднее } \\
\text { Average }\end{array}$ & $\begin{array}{c}\text { Макс. } \\
\text { Max. }\end{array}$ & $\begin{array}{c}\text { Мин. } \\
\text { Min. }\end{array}$ & $\begin{array}{c}\text { СКО } \\
\text { SD }\end{array}$ & $\begin{array}{c}\text { Средняя } \\
\text { относительная ошибка } \\
\text { Mean relative error }\end{array}$ \\
\hline$(11)$ & 0.0011 & -0.0010 & 0.2135 & -0.1775 & 0.0705 & 0.0061 \\
\hline$(8)$ & -0.0007 & 0.0017 & 0.1688 & -0.2074 & 0.0705 & 0.0062 \\
\hline$(7)$ & 0.0451 & 0.0581 & 0.5020 & -0.1718 & 0.1443 & 0.0124 \\
\hline
\end{tabular}

\section{5. Заключение}

Моделирование поведения ЖНС невозможно без тщательного учета их физических характеристик. Плотность сплавов, в силу их широкого применения в авиации, является критически важным фактором, который требует надежного и точного метода для его количественной оценки. До настоящего времени существовало некоторое количество разноплановых подходов к расчету плотности ЖНС, основанных на аналитическом, регрессионном, эмпирическом методах и их вариациях. Регрессионные методы применяются наиболее широко и дают удовлетворительные по точности результаты. Тем не менее, ни один из этих подходов не обеспечивал универсальность, достаточную для широкого применения в науке и инженерии.

В этой работе мы предложили простой эмпирикоаналитический подход и формулу расчета плотности ЖНС, основанную на этом подходе. В качестве экспериментальной проверки точности расчетов по новой формуле мы привлекли информацию по составу и плотности 69 известных ЖНС. Средняя ошибка расчета составила всего 0.001 г/ $\mathrm{cm}^{3}$.
Дополнительный материал/Supplementary material. Электронная версия статьи содержит дополнительный материал, доступный безвозмездно на сайте журнала (lettersonmaterials.com). I The online version of this paper contains supplementary material available free of charge at the journal's Web site (lettersonmaterials.com).

\section{Литература/References}

1. O. G. Ospennikova. Aviation Materials and Technologies. 1 (40), 3 (2016). (in Russian) [О.Г. Оспенникова. Авиационные материалы и технологии. 1 (40), 3 (2016).] Crossref

2. N.V. Petrushin, E. S. Elyutin, A.V. Korolev. Proceedings of the conference Fundamental and Applied Research in the Field of Creation of Cast Heat-Resistant Nickel and Intermetallide Alloys and High-efficiency Technologies for Manufacturing Garment Parts. Moscow (2017) p. 235. (in Russian) [Н.В. Петрушин, Е.С. Елютин, А.В. Королев. Сборник докладов Всероссийской научно-технической конференции Фундаментальные и прикладные исследования в области создания литейных жаропрочных никелевых и 
интерметаллидных сплавов и высокоэффективных технологий изготовления деталей газотурбинных двигателей. Москва (2017) с. 235.]

3. O.A. Bazyleva, E.G. Arginbaeva, A.V. Shestakov. Proceedings of the conference Fundamental and Applied Research in the Field of Deformable and Casting Intermetallic Alloys Based on Titanium and Nickel. Moscow (2018) p. 28. (in Russian) [O.А. Базылева, Э.Г. Аргинбаева, А.В. Шестакова. Сборник конференции Фундаментальные и прикладные исследования в области деформируемых и литейных интерметаллидных сплавов на основе титана и никеля. Москва (2018) с.28]

4. A.A. Ganeev, V.V. Smirnov, V.I. Nikitin. Metallurgy of Mechanical Engineering. 1, 42 (2020). (in Russian) [А.А. Ганеев, В.В. Смирнов, В.И. Никитин. Металлургия машиностроения. 1, 42 (2020).]

5. A.V. Logunov, Y.N. Shmotin, S.A. Zavodov, I. A. Leshchenko, D.V. Danilov, I.I. Khryashchev, A. M. Mikhaylov, A.E. Semin, M. A. Mikhaylov. Inorg. Mater: Applied Research. 7 (4), 564 (2016). ․ㅏossref

6. A.V. Logunov, I.A. Leshchenko, D.V. Danilov, I.I. Khryashchev. Journal of International Scientific Publications. 10, 58 (2016).

7. L. Zhang, Z. Huang, Y. Pan, L. Jiang. Modell. Simul. Mater. Sci. Eng. 27 (6), 065002 (2019). Crossref

8. Y. Jiang, Y.-A. Chien, C.-Y. Chen, T.-F.M. Chang, M. Sone. ECS Meeting Abstracts. MA2020-02, 1457 (2020). Crossref

9. D.E. Hooks, M. McBride, J. Stull, D. R. Johnson, E. Dervishi, R.L. Edwards. ECS Meeting Abstracts. MA2020-01, 1220 (2020). Crossref

10. F. Xiao, K. Mukai, L. Fang, Y. Fu, R.-H. Yang. T. Nonferr. Metal. Soc. 16 (6), 1263 (2006). Crossref

11. A.G. Tyagunov. Bulletin of the South Ural State University. Metallurgy Series. 16 (4), 16 (2016). (in Russian) [А.Г. Тягунов. Вестник Южно-Уральского государственного университета. Серия Металлургия. $16(4), 16(2016)$.]

12. A.G. Tyagunov, E.E. Baryshev, G.V. Tyagunov, K. Yu. Shmakova, V.S. Mushnikov. Melts. 1, 24 (2019). (in Russian) [А. Г. Тягунов, Е. Е. Барышев, Г. В. Тягунов, К. Ю. Шмакова, В.С. Мушников. Расплавы. 1, 24 (2019).]

13. R.C. Reed. The Superalloys: Fundamentals and Applications. Cambridge University Press (2006) 372 p. Crossref

14. Y. Akahama, Y. Fujimoto, T. Terai, T. Fukuda, S. Kawaguchi, N. Hirao, Y. Ohishi, T. Kakeshita. Mater. Trans. 61, 1058 (2020). Crossref

15. B. G. Livshits, V.S. Kraposhin, Ya.L. Linetskiy. Physical Properties of Metals and Alloys. Metallurgiya. Moscow. (1980) 318 p. (in Russian) [Б. Г. Лившиц, В. С. Крапошин, Я.Л. Линецкий. Физические свойства металлов и сплавов. Москва, Металлургия (1980) 318с.]

16. Web page: https://periodictable.com

17. N. V. Petrushin, I. A. Ignatova, L. A. Dyachkova. Metal Science and Heat Treatment. 9, 25 (1991). (in Russian) [Н.В. Петрушин, И.А. Игнатова, Л.А. Дьячкова. Металловедение и термическая обработка металлов. 9, 25 (1991).]

18. A. V. Logunov, Yu. N. Shmotin, D. V. Danilov. Metal Technology. 7, 3 (2014). (in Russian) [A.В. Логунов, Ю.Н. Шмотин, Д. В. Данилов. Технология Металлов. 7, 3 (2014).]

19. R. Parsons, K. Ono, Z. Li, H. Kishimoto, T. Shoji, A. Kato, M. R. Hill, K. Suzuki. J. All. Compd. 859, 157845 (2020). Crossref

20. A.A. Glotka, S. V. Haiduk. Science and progress to transport. 2 (80), 91 (2019). (in Russian) [А.А. Глотка, С. В. Гайдук. Наука та прогрес транспорту. 2 (80), 91 (2019).]

21. Web page: https://www.handymath.com/calculators.html 22. F. C. Hull. Met. Prog. 5 (96), 139 (1969). 